\title{
The Bifurcations of Traveling Wave Solutions of the Kundu Equation
}

\author{
Yating Yi and Zhengrong Liu \\ Department of Mathematics, South China University of Technology, Guangzhou 510640, China \\ Correspondence should be addressed to Yating Yi; yi.yating@mail.scut.edu.cn
}

Received 12 July 2013; Accepted 20 August 2013

Academic Editor: Michael Meylan

Copyright (C) 2013 Y. Yi and Z. Liu. This is an open access article distributed under the Creative Commons Attribution License, which permits unrestricted use, distribution, and reproduction in any medium, provided the original work is properly cited.

\begin{abstract}
We use the bifurcation method of dynamical systems to study the bifurcations of traveling wave solutions for the Kundu equation. Various explicit traveling wave solutions and their bifurcations are obtained. Via some special phase orbits, we obtain some new explicit traveling wave solutions. Our work extends some previous results.
\end{abstract}

\section{Introduction}

In this paper, we consider the bifurcations of traveling wave solutions for the Kundu equation with the fifth-order nonlinear term:

$$
\begin{aligned}
i u_{t}+ & u_{x x}+c_{3}|u|^{2} u+c_{5}|u|^{4} u \\
& -i s_{2}\left(|u|^{2} u\right)_{x}-i r\left(|u|^{2}\right)_{x} u=0, \quad x \in R,
\end{aligned}
$$

where $c_{3}, c_{5}, s_{2}$, and $r$ are real constants. Equation (1a) was derived by Kundu [1] in the study of integrability, and it is an important special case of the generalized complex GinzburgLandau [2].

For convenience, we denote the Kundu equation by

$$
\begin{aligned}
u_{t}= & i u_{x x}+i\left(c_{3}|u|^{2}+c_{5}|u|^{4}\right) u \\
& +\alpha|u|^{2} u_{x}+\beta u^{2} \bar{u}_{x}, \quad x \in R,
\end{aligned}
$$

where $\alpha=2 s_{2}+r, \beta=s_{2}+r$.

Meanwhile, (1a) and (1b) and their special cases arise in various physical and mechanical applications, such as plasma physics, nonlinear fluid mechanics, nonlinear optics, and quantum physics (see [2-7]). For example, (1b) contains the following particular cases.

(1) If $r=0$, then (la) and (lb) reduce to the derivative Schrödinger equation:

$$
u_{t}=i u_{x x}+i\left(c_{3}|u|^{2}+c_{5}|u|^{4}\right) u+s_{2}\left(|u|^{2} u\right)_{x} .
$$

(2) If $c_{3}=c_{5}=0, s_{2}=-\delta$, and $r=-s_{2}$, then (1a) and (1b) reduce to Chen-Lee-Liu equation [8]:

$$
i u_{t}+u_{x x}+i \delta|u|^{2} u_{x}=0 .
$$

(3) If $c_{5}=2 \delta^{2}, s_{2}=2 \delta$, and $r=-2 s_{2}$, then (1a) and (1b) reduce to Gerdjikov-Ivanov equation [9]:

$$
i u_{t}+u_{x x}+c_{3}|u|^{2} u+2 \delta^{2}|u|^{4} u+2 i \delta u^{2} \bar{u}_{x}=0 .
$$

In [2], by using primary integral method, van Saarloos and Hohenberg obtained the exact solitary waves of the form

$$
\begin{array}{r}
u(x, t)=e^{-i \omega t} e^{i \psi(x-c t)} \varphi(x-c t)=e^{-i \omega t} e^{i \psi(\xi)} \varphi(\xi), \\
\xi=x-c t,
\end{array}
$$

for (2). Furthermore, Biswas [10] carried out the integration of the generalized Kundu equation to obtain the 1-soliton solution. Feng and Wang [11] gave the explicit solitary wave solutions by the algebraic curve method for (1a) and (1b). Bright/dark solitary wave solutions and triangular periodic wave solutions of (1a) and (1b) were obtained in [12]. Zhang et al. [13] obtained exact solitary waves of (1a) and (1b) by using the undetermined coefficient method, and they also investigated orbital stability of solitary waves. Zhang [14] obtained various exact traveling wave solutions of (1a) and (lb) by the general solutions of a kind of subequation. Besides, Guo and Wu proved that the kind of solitary waves (5) for 
(2) is stable in [15]. He and Meng [16] investigated the exact traveling waves for (4) by using the bifurcation theory and the method of phase portraits analysis.

In this paper, we use the bifurcation method of dynamical systems [17-23] to study the traveling wave solutions and their bifurcations for (1a) and (1b). Through bifurcation phase portraits, we obtain various traveling wave solutions of (1a) and (1b). Via some special phase orbits, we also obtain new explicit traveling wave solutions. Not only the existence of these solutions is proved, but also their concrete expressions are presented. Our work extends some previous results [10$16]$.

In order to derive traveling wave solutions, we assume that (1a) and (1b) have solutions of the form (5). Substituting (5) into (1a) and (1b) and making the real part and imaginary part of (la) and (1b) equal to zero, we have

$$
\begin{aligned}
\psi^{\prime \prime} \varphi+2 \psi^{\prime} \varphi^{\prime}-\alpha \varphi^{2} \varphi^{\prime}-\beta \varphi^{2} \varphi^{\prime}-c \varphi^{\prime} & =0, \\
\varphi^{\prime \prime}+\left[c_{3} \varphi^{2}+c_{5} \varphi^{4}+\alpha \psi^{\prime} \varphi^{2}\right. & \\
\left.-\beta \psi^{\prime} \varphi^{2}-\left(\psi^{\prime}\right)^{2}+\omega+c \psi^{\prime}\right] \varphi & =0 .
\end{aligned}
$$

Let

$$
\psi^{\prime}(\xi)=E+D \varphi^{2}(\xi)
$$

Substituting (8) into (6) and equating the coefficients of these terms $\varphi, \varphi^{\prime}$, and $\varphi^{\prime \prime}$ to zero, we get $E=c / 2, D=(\alpha+$ $\beta) / 4$. Therefore, when

$$
\psi^{\prime}(\xi)=\frac{c}{2}+\frac{\alpha+\beta}{4} \varphi^{2}(\xi)
$$

(6) is identical to zero. Combining (9) and (7), we obtain the following equation:

$$
\varphi^{\prime \prime}-d_{1} \varphi-2 d_{2} \varphi^{3}-3 d_{4} \varphi^{5}=0,
$$

where

$$
\begin{gathered}
d_{1}=-\omega-\frac{c^{2}}{4}, \\
d_{2}=-\frac{c_{3}}{2}-\frac{(\alpha-\beta) c}{4}, \\
d_{4}=-\frac{1}{3}\left[c_{5}+\frac{(\alpha+\beta)(3 \alpha-5 \beta)}{16}\right] .
\end{gathered}
$$

In fact, (10) is equivalent to the system

$$
\begin{gathered}
\frac{d \varphi}{d \xi}=y, \\
\frac{d y}{d \xi}=d_{1} \varphi+2 d_{2} \varphi^{3}+3 d_{4} \varphi^{5},
\end{gathered}
$$

which has the first integral

$$
H(\varphi, y)=y^{2}-d_{1} \varphi^{2}-d_{2} \varphi^{4}-d_{4} \varphi^{6}=h .
$$

The rest of the paper is organized as follows. Our main results and remarks are arranged in Section 2. In Section 3, in order to derive the main results, we show various planar systems and their bifurcation phase portraits of (10). We state the theoretic derivation of our main results by the bifurcation method of dynamical systems in Section 4. Short conclusions are given in Section 5.

\section{Main Results}

In this section, we state our main results and give some corresponding remarks. Our main results are listed in the following two propositions.

Proposition 1. When the parameters $d_{4}>0$ and $d_{2}<0$, (10) possesses the following solutions.

(1) If $d_{1}=d_{2}^{2} / 3 d_{4}$, the blowup solution is of expression

$$
\varphi_{1}(\xi)=\frac{\sqrt{\left(-d_{2} / 3 d_{4}\right)\left(\left(d_{2} / 3 \sqrt{d_{4}}\right)|\xi|+1\right)}}{\sqrt{\left(\left(d_{2} / 3 \sqrt{d_{4}}\right)|\xi|+1\right)^{2}-1}} .
$$

(2) If $d_{2}^{2} / 4 d_{4}<d_{1}<d_{2}^{2} / 3 d_{4}$, the blowup solution is of expression

$$
\varphi_{2}(\xi)=\frac{a_{1} a_{2} \tanh \eta_{1}}{\sqrt{a_{1} \tanh ^{2} \eta_{1}+a_{2}^{2}-a_{1}^{2}}}
$$

and the solitary wave solution possesses the expression

$$
\varphi_{3}(\xi)=\frac{a_{1} a_{2} \tanh \eta_{2}}{\sqrt{a_{1} \tanh ^{2} \eta_{2}+a_{2}^{2}-a_{1}^{2}}},
$$

where

$$
a_{1}=\sqrt{\frac{1}{3}\left[-\frac{d_{2}}{d_{4}}-\sqrt{\left.\frac{d_{2}^{2}}{d_{4}^{2}}-3 \frac{d_{1}}{d_{4}}\right]}\right.},
$$

$$
\begin{gathered}
a_{2}=\sqrt{\frac{1}{3}\left[-\frac{d_{2}}{d_{4}}+2 \sqrt{\left.\frac{d_{2}^{2}}{d_{4}^{2}}-3 \frac{d_{1}}{d_{4}}\right]},\right.} \\
\eta_{1}=-a_{1} \sqrt{a_{1}^{2}-a_{2}^{2}} \sqrt{d_{4}}|\xi|+\operatorname{arctanh} \frac{\sqrt{a_{1}^{2}-a_{2}^{2}}}{a_{1}}, \\
\eta_{2}=a_{1} \sqrt{a_{1}^{2}-a_{2}^{2}} \sqrt{d_{4}}|\xi|+\frac{\pi}{2} .
\end{gathered}
$$

(3) If $d_{1}=d_{2}^{2} / 4 d_{4}$, the blowup solution is of expression

$$
\varphi_{4}(\xi)=\frac{\varphi_{2}}{\sqrt{1-e^{-2 \varphi_{2}^{2} \sqrt{d_{4}}|\xi|}}}
$$

the kink wave solution is of expression

$$
\varphi_{5}(\xi)=\frac{\varphi_{2}}{\sqrt{1+\left(\left(\varphi_{2}^{2}-\varphi_{3}^{2}\right) / \varphi_{3}^{2}\right) e^{-2 \varphi_{2}^{2} \sqrt{d_{4}} \xi}}},
$$


and the periodic blowup solution is of expression

$$
\varphi_{6}(\xi)=\sqrt{\varphi_{3}^{2}+\frac{2 \varphi_{3}^{2}\left(\varphi_{3}^{2}-\varphi_{1}^{2}\right)}{\varphi_{1}^{2} \sin \left(c_{1}-\eta_{3}\right)-2 \varphi_{3}^{2}+\varphi_{1}^{2}}},
$$

where

$$
\begin{gathered}
\varphi_{1}=\sqrt{-\frac{2 d_{2}}{3 d_{4}}}, \\
\varphi_{2}=\sqrt{-\frac{d_{2}}{2 d_{4}}}, \\
\varphi_{3}=\sqrt{-\frac{d_{2}}{6 d_{4}}}, \\
c_{1}=\arcsin \frac{2 \varphi_{3}^{2}-\varphi_{1}^{2}}{\varphi_{1}^{2}}, \\
\eta_{3}=2 \varphi_{3} \sqrt{\varphi_{1}^{2}-\varphi_{3}^{2}} \sqrt{d_{4}}|\xi| .
\end{gathered}
$$

(4) If $0<d_{1}<d_{2}^{2} / 4 d_{4}$, the solitary wave solution is of expression

$$
\varphi_{7}(\xi)=\frac{\sqrt{2} a_{3} a_{4}}{\sqrt{a_{3}^{2}+a_{4}^{2}+\left(a_{3}^{2}-a_{4}^{2}\right) \cosh \eta_{4}}},
$$

and the kink wave solution is of expression

$$
\varphi_{8}(\xi)=\frac{a_{5} a_{6} \tanh \eta_{5}}{\sqrt{a_{5}^{2}+a_{6}^{2}+a_{5}^{2} \tanh ^{2} \eta_{5}}},
$$

where

$$
\begin{gathered}
a_{3}=\sqrt{\frac{-d_{2}+\sqrt{d_{2}^{2}-4 d_{1} d_{4}}}{2 d_{4}}}, \\
a_{4}=\sqrt{\frac{-d_{2}-\sqrt{d_{2}^{2}-4 d_{1} d_{4}}}{2 d_{4}}}, \\
a_{5}=\sqrt{\frac{-d_{2}+\sqrt{d_{2}^{2}-4 d_{1} d_{4}}}{3 d_{4}}}, \\
a_{6}=\sqrt{\frac{d_{2}+2 \sqrt{d_{2}^{2}-4 d_{1} d_{4}}}{3 d_{4}}}, \\
\eta_{4}=-2 a_{3} a_{4} \sqrt{d_{4}} \xi, \\
\eta_{5}=a_{5} \sqrt{a_{5}^{2}+a_{6}^{2}} \sqrt{d_{4}} \xi .
\end{gathered}
$$

(5) If $d_{1}=0$, the blowup solutions are of expressions

$$
\begin{gathered}
\varphi_{9}(\xi)=\frac{1}{\sqrt{d_{2} \xi^{2}-2 \sqrt{d_{4}} \xi}}, \\
\varphi_{10}(\xi)=\frac{\sqrt{-\left(2 d_{2} / d_{4}\right)} \tanh \eta_{6}}{3-2 \tanh ^{2} \eta_{6}},
\end{gathered}
$$

and the kink wave solution is of expression

$$
\varphi_{11}(\xi)=\frac{\sqrt{-\left(2 d_{2} / d_{4}\right)} \tanh \eta_{7}}{3-2 \tanh ^{2} \eta_{7}}
$$

where

$$
\begin{gathered}
\eta_{6}=\operatorname{arctanh} \frac{\sqrt{6}}{2}+\frac{\sqrt{6}}{3} \frac{d_{2}}{\sqrt{d_{4}}}|\xi|, \\
\eta_{7}=-\frac{\sqrt{6}}{3} \frac{d_{2}}{\sqrt{d_{4}}} \xi .
\end{gathered}
$$

Proposition 2. When the parameters $d_{4}<0$ and $d_{2}>0$, (10) possesses the following solutions.

(1) If $d_{1}=d_{2}^{2} / 3 d_{4}$, the solitary wave solution is of expression

$$
\varphi_{12}(\xi)=\sqrt{\frac{d_{2}^{2} \xi^{2}}{27 d_{4}^{2}-3 d_{2}^{2} d_{4} \xi^{2}}}
$$

(2) If $d_{2}^{2} / 3 d_{4}<d_{1}<d_{2}^{2} / 4 d_{4}$, the solitary wave solution is of expression

$$
\varphi_{13}(\xi)=\frac{a_{7} a_{8}\left(1+e^{\eta_{8}}\right)}{\sqrt{4 a_{8}^{2} e^{\eta_{8}}+a_{7}^{2}\left(1-e^{\eta_{8}}\right)^{2}}},
$$

the kink wave solution is of expression

$$
\varphi_{14}(\xi)=\frac{a_{7} a_{8}\left(e^{\eta_{9}}-1\right)}{\sqrt{a_{7}^{2}\left(1+e^{\eta_{9}}\right)^{2}-4 a_{8}^{2} e^{\eta_{9}}}},
$$

and the periodic wave solution is of expression

$$
\varphi_{15}(\xi)=\frac{a_{9} a_{10} \tan \eta_{10}}{\sqrt{a_{9}^{2}-a_{10}^{2}+a_{9}^{2} \tan ^{2} \eta_{10}}},
$$


where

$$
\begin{gathered}
a_{7}=\sqrt{-\frac{1}{3}\left[\frac{d_{2}}{d_{4}}-2 \sqrt{\left.\frac{d_{2}^{2}}{d_{4}^{2}}-3 \frac{d_{1}}{d_{4}}\right]}\right.}, \\
a_{8}=\sqrt{-\frac{1}{3}\left[\frac{d_{2}}{d_{4}}-\sqrt{\left.\frac{d_{2}^{2}}{d_{4}^{2}}-3 \frac{d_{1}}{d_{4}}\right]}\right.} \\
a_{9}=\sqrt{\frac{1}{3}\left[-\frac{d_{2}}{d_{4}}+\sqrt{\left.\frac{d_{2}^{2}}{d_{4}^{2}}-3 \frac{d_{1}}{d_{4}}\right]}\right.} \\
a_{10}=\sqrt{\frac{1}{3}\left[-\frac{d_{2}}{d_{4}}-2 \sqrt{\frac{d_{2}^{2}}{d_{4}^{2}}-3 \frac{d_{1}}{d_{4}}}\right]} \\
\eta_{10}=-2 a_{8} \sqrt{a_{7}^{2}-a_{8}^{2}} \sqrt{-d_{4}}|\xi| \\
\eta_{9}=2 a_{8} \sqrt{a_{7}^{2}-a_{8}^{2}} \sqrt{-d_{4}^{2}} \xi \\
\eta_{10}^{2} \sqrt{-d_{4}}|\xi|
\end{gathered}
$$

(3) If $d_{1}=d_{2}^{2} / 4 d_{4}$, the solitary wave solution is of expression

$$
\varphi_{16}(\xi)=\frac{\sqrt{-\left(d_{2} / 3 d_{4}\right)}\left(1+e^{\eta_{11}}\right)}{\sqrt{2 e^{\eta_{11}}+2\left(1-e^{\eta_{11}}\right)^{2}}},
$$

and the kink wave solution is of expression

$$
\varphi_{17}(\xi)=\frac{\sqrt{-\left(d_{2} / 3 d_{4}\right)}\left(e^{\eta_{12}}-1\right)}{\sqrt{2\left(1+e^{\eta_{12}}\right)^{2}-2 e^{\eta_{12}}}}
$$

where

$$
\begin{gathered}
\eta_{11}=-\frac{d_{2}}{\sqrt{-3 d_{4}}}|\xi| \\
\eta_{12}=\frac{d_{2}}{\sqrt{-3 d_{4}}} \xi
\end{gathered}
$$

(4) If $d_{2}^{2} / 4 d_{4}<d_{1}<0$, the periodic wave solution is of expression

$$
\varphi_{18}(\xi)=\frac{\sqrt{2} a_{11} a_{12}}{\sqrt{a_{11}^{2}+a_{12}^{2}+\left(a_{11}^{2}-a_{12}^{2}\right) \cos \eta_{13}}}
$$

where

$$
\begin{gathered}
a_{11}=\sqrt{\frac{1}{2}\left[-\frac{d_{2}}{d_{4}}-\frac{\sqrt{d_{2}^{2}-4 d_{1} d_{4}}}{d_{4}}\right]}, \\
a_{12}=\sqrt{\frac{1}{2}\left[-\frac{d_{2}}{d_{4}}+\frac{\sqrt{d_{2}^{2}-4 d_{1} d_{4}}}{d_{4}}\right]}, \\
\eta_{13}=2 a_{11} a_{12} \sqrt{-d_{4}}|\xi| .
\end{gathered}
$$

(5) If $d_{1}=0$, the solitary wave solution is of expression

$$
\varphi_{19}(\xi)=\frac{\sqrt{d_{2}}}{\sqrt{-d_{4}+d_{2}^{2} \xi^{2}}} .
$$

Remark 1. The traveling wave solutions $\varphi_{i}(\xi)(i=3,5,6,7,9$, $11,14,15,18,19)$ are the same as the ones in $[10-14]$, while the rest of the solutions are new, which cannot be found in the literature.

Remark 2. Choosing

$$
d_{4}=\frac{1}{16}, \quad d_{2}=-\frac{1}{4}
$$

we can obtain all the exact solutions reported in [16].

Remark 3. The correctness of the above solutions is tested by using the software Mathematica.

\section{The Bifurcation Phase Portraits}

In order to derive the main results mentioned in Section 2, we draw the bifurcation phase portraits of system (12) by the qualitative theory of dynamical systems.

First, letting

$$
f(\varphi)=d_{1} \varphi+2 d_{2} \varphi^{3}+3 d_{4} \varphi^{5}
$$

we have

$$
f^{\prime}(\varphi)=d_{1}+6 d_{2} \varphi^{2}+15 d_{4} \varphi^{4}
$$


Solving $f(\varphi)=0$, we get five roots as follows:

$$
\begin{gathered}
\varphi^{*}=0, \\
\varphi_{1}^{+}=\sqrt{\frac{-d_{2}+\sqrt{d_{2}^{2}-3 d_{1} d_{4}}}{3 d_{4}}}, \\
\varphi_{1}^{-}=-\sqrt{\frac{-d_{2}+\sqrt{d_{2}^{2}-3 d_{1} d_{4}}}{3 d_{4}}}, \\
\varphi_{2}^{+}=\sqrt{\frac{-d_{2}-\sqrt{d_{2}^{2}-3 d_{1} d_{4}}}{3 d_{4}}}, \\
\varphi_{2}^{-}=-\sqrt{\frac{-d_{2}-\sqrt{d_{2}^{2}-3 d_{1} d_{4}}}{3 d_{4}}} .
\end{gathered}
$$

Second, let $(\varphi, 0)$ be one of the singular points of system (12). The characteristic values of the linearized system of system $(12)$ at $(\varphi, 0)$ are

$$
\lambda_{ \pm}(\varphi, 0)= \pm \sqrt{f^{\prime}(\varphi)} .
$$

From the qualitative theory of dynamical systems, we get the properties of the singular point $(\varphi, 0)$ as follows.

(i) If $f^{\prime}(\varphi)>0$, then $(\varphi, 0)$ is a saddle point of system (12).

(ii) If $f^{\prime}(\varphi)<0$, then $(\varphi, 0)$ is a center point of system (12).

(iii) If $f^{\prime}(\varphi)=0$, then $(\varphi, 0)$ is a degenerate singular point of system (12).

By using the property of equilibrium points and bifurcation theory, we obtain four bifurcation curves in the $\left(d_{2}, d_{1}\right)$ parameter plane as follows:

$$
\begin{array}{ll}
l_{1}: d_{1}=\frac{d_{2}^{2}}{3 d_{4}}, & l_{2}: d_{1}=\frac{d_{2}^{2}}{4 d_{4}} \quad\left(d_{4}>0\right), \\
l_{3}: d_{1}=\frac{d_{2}^{2}}{3 d_{4}}, & l_{4}: d_{1}=\frac{d_{2}^{2}}{4 d_{4}} \quad\left(d_{4}<0\right) .
\end{array}
$$

Then, according to the qualitative theory, we obtain the bifurcation phase portraits of system (12) as shown in Figures 1 and 2 .

\section{The Derivations for Proposition 1}

In this section, by using the above bifurcation phase portraits (Figure 1) and (12) and (13), we will give the exact traveling wave solutions of (10) under the given parameter conditions shown in Section 2.

(1) When $d_{1}=d_{2}^{2} / 3 d_{4}$, the curve $\Gamma_{1}$ possesses the following expression:

$$
y^{2}=d_{4}\left(\varphi^{2}-\varphi_{0}^{2}\right)^{3}, \quad \text { where } \varphi \geq \varphi_{0},
$$

where $\varphi_{0}=\sqrt{-d_{2} / 3 d_{4}}$. Substituting (45) into $d \varphi / d \xi=y$ and integrating it along $\Gamma_{1}$, we have

$$
\int_{\varphi}^{+\infty} \frac{d s}{\left(s^{2}-\varphi_{0}^{2}\right) \sqrt{s^{2}-\varphi_{0}^{2}}}=-\sqrt{d_{4}}|\xi| .
$$

In (46) completing the integral and solving the equation for $\varphi$, we get the solution $\varphi_{1}(\xi)$.

(2) When $d_{2}^{2} / 4 d_{4}<d_{1}<d_{2}^{2} / 3 d_{4}$, the curves $\Gamma_{2}$ and $\Gamma_{3}$ have the following expressions:

$$
\begin{gathered}
\Gamma_{2}: y^{2}=d_{4}\left(\varphi^{2}-a_{1}^{2}\right)^{2}\left(\varphi^{2}-a_{2}^{2}\right), \quad \text { where } a_{1}<\varphi, \\
\Gamma_{3}: y^{2}=d_{4}\left(a_{1}^{2}-\varphi^{2}\right)^{2}\left(\varphi^{2}-a_{2}^{2}\right), \quad \text { where } a_{2} \leq \varphi<a_{1} .
\end{gathered}
$$

Substituting (47) into $d \varphi / d \xi=y$ and integrating them along $\Gamma_{2}$ and $\Gamma_{3}$, respectively, it follows that

$$
\begin{aligned}
& \int_{\varphi}^{+\infty} \frac{d s}{\left(s^{2}-a_{1}^{2}\right) \sqrt{s^{2}-a_{2}^{2}}}=-\sqrt{d_{4}}|\xi| \quad\left(\operatorname{along} \Gamma_{2}\right), \\
& \int_{a_{2}}^{\varphi} \frac{d s}{\left(a_{1}^{2}-s^{2}\right) \sqrt{s^{2}-a_{2}^{2}}}=-\sqrt{d_{4}}|\xi| \quad\left(\operatorname{along} \Gamma_{3}\right) .
\end{aligned}
$$

Completing the integrals above and solving them for $\varphi$, we obtain the solutions $\varphi_{2}(\xi)$ and $\varphi_{3}(\xi)$.

(3) When $d_{1}=d_{2}^{2} / 4 d_{4}$, the curves $\Gamma_{4}, \Gamma_{5}$, and $\Gamma_{6}$ have the following expressions:

$$
\Gamma_{4}: y^{2}=d_{4}\left(\varphi^{2}-\varphi_{2}^{2}\right)^{2} \varphi^{2}, \quad \text { where } \varphi_{2}<\varphi \text {, }
$$

$\Gamma_{5}: y^{2}=d_{4}\left(\varphi_{2}^{2}-\varphi^{2}\right)^{2} \varphi^{2}, \quad$ where $0<\varphi<\varphi_{2}$,

$\Gamma_{6}: y^{2}=d_{4}\left(\varphi^{2}-\varphi_{3}^{2}\right)^{2}\left(\varphi^{2}-\varphi_{1}^{2}\right), \quad$ where $\varphi_{1} \leq \varphi$.

Substituting (49) into $d \varphi / d \xi=y$ and integrating them along $\Gamma_{4}, \Gamma_{5}$, and $\Gamma_{6}$, respectively, it follows that

$$
\begin{gathered}
\int_{\varphi}^{+\infty} \frac{d s}{\left(s^{2}-\varphi_{2}^{2}\right) s}=\sqrt{d_{4}}|\xi| \quad\left(\text { along } \Gamma_{4}\right), \\
\int_{\varphi_{3}}^{\varphi} \frac{d s}{\left(\varphi_{2}^{2}-s^{2}\right) s}=\sqrt{d_{4}} \xi \quad\left(\text { along } \Gamma_{5}\right), \\
\int_{\varphi}^{+\infty} \frac{d s}{\left(s^{2}-\varphi_{3}^{2}\right) \sqrt{s^{2}-\varphi_{1}^{2}}}=\sqrt{d_{4}}|\xi| \quad\left(\text { along } \Gamma_{6}\right) .
\end{gathered}
$$

Completing the integrals above, we obtain the solutions $\varphi_{i}(\xi)(i=4,5,6)$. 


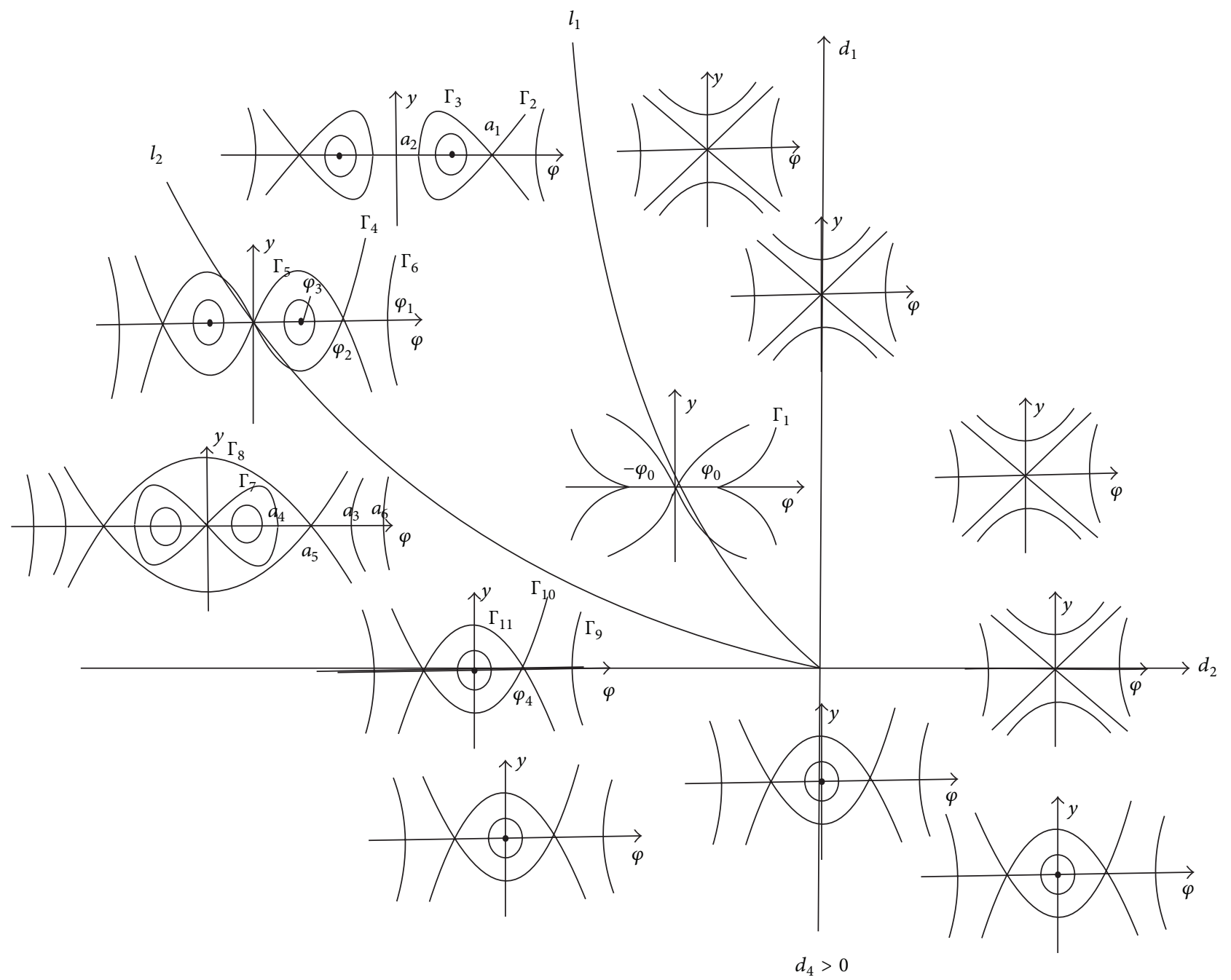

FIGURE 1: The phase portraits of system (12) when $d_{4}>0$.

(4) When $0<d_{1}<d_{2}^{2} / 4 d_{4}$, the curves $\Gamma_{7}$ and $\Gamma_{8}$ have the following expressions:

$$
\begin{array}{r}
\Gamma_{7}: y^{2}=d_{4}\left(a_{3}^{2}-\varphi^{2}\right)\left(a_{4}^{2}-\varphi^{2}\right) \varphi^{2}, \\
\text { where } 0<\varphi \leq a_{4}, \\
\Gamma_{8}: y^{2}=d_{4}\left(a_{5}^{2}-\varphi^{2}\right)^{2}\left(\varphi^{2}+a_{6}^{2}\right), \\
\text { where }-a_{5}<\varphi<a_{5} .
\end{array}
$$

Substituting (51) into $d \varphi / d \xi=y$ and integrating them along $\Gamma_{7}$ and $\Gamma_{8}$, respectively, it follows that

$$
\begin{gathered}
\int_{a_{4}}^{\varphi} \frac{d s}{s \sqrt{\left(a_{3}^{2}-s^{2}\right)\left(a_{4}^{2}-s^{2}\right)}}=\sqrt{d_{4}}|\xi| \quad\left(\text { along } \Gamma_{7}\right), \\
\int_{0}^{\varphi} \frac{d s}{\left(a_{5}^{2}-s^{2}\right) \sqrt{s^{2}+a_{6}^{2}}}=\sqrt{d_{4}} \xi \quad\left(\text { along } \Gamma_{8}\right) .
\end{gathered}
$$

Completing the integrals above, we obtain the solutions $\varphi_{7}(\xi)$ and $\varphi_{8}(\xi)$.

(5) If $d_{1}=0$, the curves $\Gamma_{9}, \Gamma_{10}$, and $\Gamma_{11}$ have the following expressions:

$$
\Gamma_{9}: y^{2}=d_{4} \varphi^{6}+d_{2} \varphi^{4}, \quad \text { where } \sqrt{\frac{-d_{2}}{d_{4}}} \leq \varphi,
$$

$$
\Gamma_{10}: y^{2}=d_{4}\left(\varphi^{2}-\varphi_{4}^{2}\right)^{2}\left(\varphi^{2}-\frac{d_{2}}{3 d_{4}}\right)
$$

$$
\text { where } \varphi>\varphi_{4}=\sqrt{\frac{-2 d_{2}}{3 d_{4}}} \text {, }
$$

$$
\Gamma_{11}: y^{2}=d_{4}\left(\varphi_{4}^{2}-\varphi^{2}\right)^{2}\left(\varphi^{2}-\frac{d_{2}}{3 d_{4}}\right)
$$

where $-\varphi_{4}<\varphi<\varphi_{4}$. 


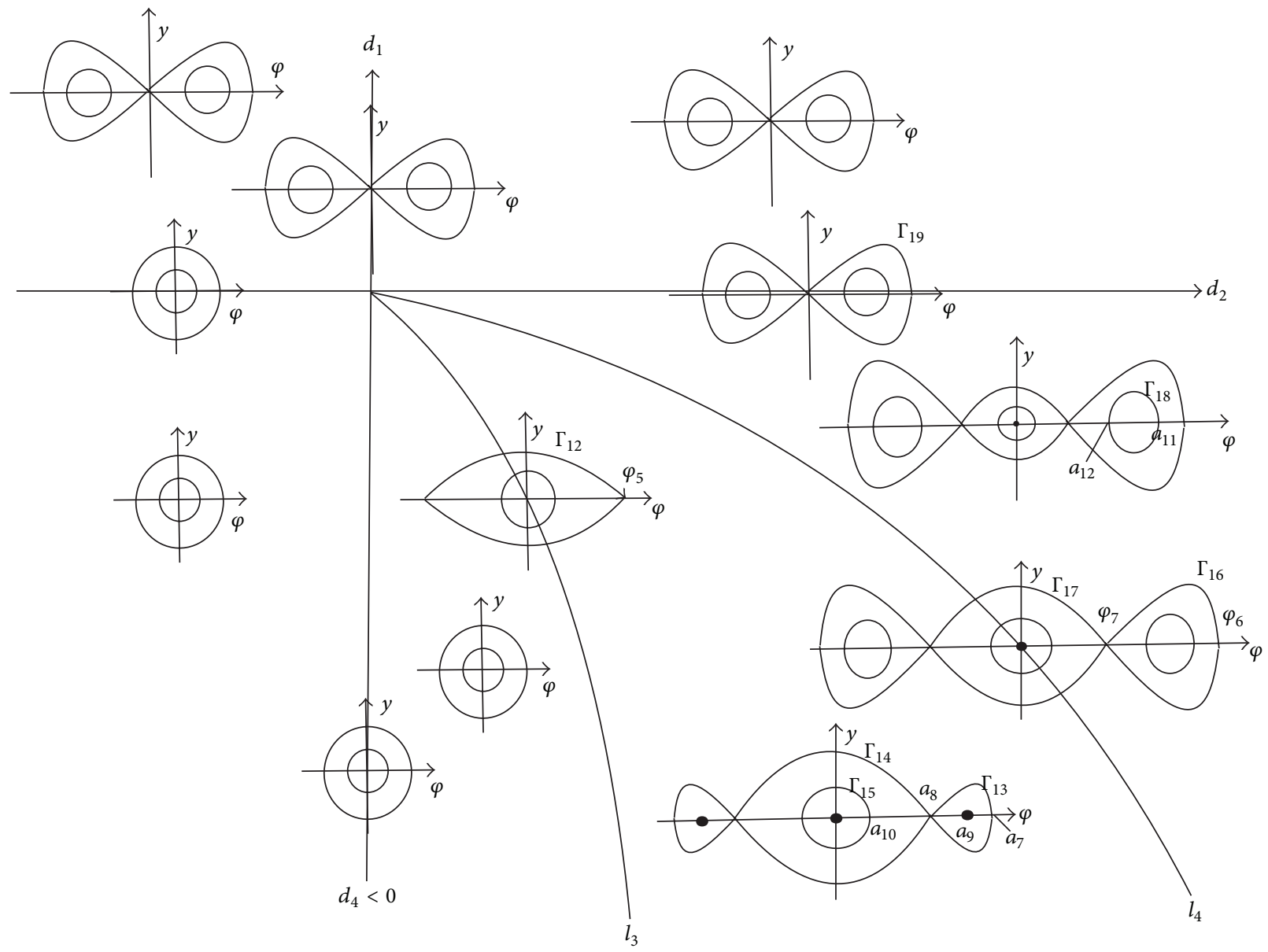

Figure 2: The phase portraits of system (12) when $d_{4}<0$.

Substituting (53) into $d \varphi / d \xi=y$ and integrating them along $\Gamma_{9}, \Gamma_{10}$, and $\Gamma_{11}$, respectively, it follows that

$$
\begin{gathered}
\int_{\varphi}^{+\infty} \frac{d s}{s^{2} \sqrt{s^{2}+d_{2} / d_{4}}}=-\sqrt{d_{4}}|\xi| \quad\left(\text { along } \Gamma_{9}\right), \\
\int_{\varphi}^{+\infty} \frac{d s}{\left(s^{2}-\varphi_{4}^{2}\right) \sqrt{s^{2}-d_{2} / 3 d_{4}}}=-\sqrt{d_{4}}|\xi| \quad\left(\text { along } \Gamma_{10}\right), \\
\int_{0}^{\varphi} \frac{d s}{\left(\varphi_{4}^{2}-s^{2}\right) \sqrt{s^{2}-d_{2} / 3 d_{4}}}=\sqrt{d_{4}} \xi \quad\left(\text { along } \Gamma_{11}\right) .
\end{gathered}
$$

Completing the integrals above, we obtain the solutions $\varphi_{i}(\xi)(i=9,10,11)$ and complete the derivations for Proposition 1.

\section{The Derivations for Proposition 2}

Similarly, we will complete the derivations for Proposition 2 by using the bifurcation phase portraits (Figure 2) and (12) and (13).
(1) When $d_{1}=d_{2}^{2} / 3 d_{4}$, the curve $\Gamma_{12}$ possesses the following expression:

$$
y^{2}=-d_{4}\left(\varphi_{5}^{2}-\varphi^{2}\right)^{3}
$$

where $\varphi_{5}=\sqrt{-d_{2} / 3 d_{4}}$ and $-\varphi_{5}<\varphi<\varphi_{5}$. Substituting (55) into $d \varphi / d \xi=y$ and integrating it along $\Gamma_{12}$, we have

$$
\int_{0}^{\varphi} \frac{d s}{\left(\varphi_{5}^{2}-s^{2}\right) \sqrt{\varphi_{5}^{2}-s^{2}}}=\sqrt{-d_{4}}|\xi| .
$$

In (56) completing the integral and solving the equation for $\varphi$, we get the solution $\varphi_{12}(\xi)$.

(2) When $d_{2}^{2} / 3 d_{4}<d_{1}<d_{2}^{2} / 4 d_{4}$, the curves $\Gamma_{13}, \Gamma_{14}$, and $\Gamma_{15}$ have the following expressions:

$$
\begin{array}{r}
\Gamma_{13}: y^{2}=-d_{4}\left(a_{7}^{2}-\varphi^{2}\right)\left(\varphi^{2}-a_{8}^{2}\right)^{2}, \\
\text { where } a_{8}<\varphi \leq a_{7},
\end{array}
$$




$$
\begin{array}{r}
\Gamma_{14}: y^{2}=-d_{4}\left(a_{8}^{2}-\varphi^{2}\right)^{2}\left(a_{7}^{2}-\varphi^{2}\right), \\
\text { where }-a_{8}<\varphi<a_{8}, \\
\Gamma_{15}: y^{2}=-d_{4}\left(a_{9}^{2}-\varphi^{2}\right)^{2}\left(a_{10}^{2}-\varphi^{2}\right), \\
\text { where }-a_{10} \leq \varphi \leq a_{10} .
\end{array}
$$

Substituting (57) into $d \varphi / d \xi=y$ and integrating them along $\Gamma_{13}, \Gamma_{14}$, and $\Gamma_{15}$, respectively, it follows that

$$
\begin{aligned}
& \int_{a_{7}}^{\varphi} \frac{d s}{\left(s^{2}-a_{8}^{2}\right) \sqrt{a_{7}^{2}-s^{2}}}=\sqrt{-d_{4}}|\xi| \quad\left(\operatorname{along} \Gamma_{13}\right), \\
& \int_{0}^{\varphi} \frac{d s}{\left(a_{8}^{2}-s^{2}\right) \sqrt{a_{7}^{2}-s^{2}}}=\sqrt{-d_{4}}|\xi| \quad\left(\operatorname{along} \Gamma_{14}\right), \\
& \int_{0}^{\varphi} \frac{d s}{\left(a_{9}^{2}-s^{2}\right) \sqrt{a_{10}^{2}-s^{2}}}=\sqrt{-d_{4}}|\xi| \quad\left(\text { along } \Gamma_{15}\right) .
\end{aligned}
$$

Completing the integrals above, we obtain the solutions $\varphi_{i}(\xi)(i=13,14,15)$.

(3) When $d_{1}=d_{2}^{2} / 4 d_{4}$, the curves $\Gamma_{16}$, and $\Gamma_{17}$ have the following expressions:

$$
\Gamma_{16}: y^{2}=-d_{4}\left(\varphi^{2}-\varphi_{7}^{2}\right)^{2}\left(\varphi_{6}^{2}-\varphi^{2}\right),
$$

where $\varphi_{6}=\sqrt{-2 d_{2} / 3 d_{4}}, \varphi_{7}=\sqrt{-d_{2} / 6 d_{4}}$ and $\varphi_{7}<\varphi \leq \varphi_{6}$,

$$
\begin{array}{r}
\Gamma_{17}: y^{2}=-d_{4}\left(a_{7}^{2}-\varphi^{2}\right)^{2}\left(a_{6}^{2}-\varphi^{2}\right), \\
\text { where }-a_{7}<\varphi<a_{7} .
\end{array}
$$

Substituting (59) and (60) into $d \varphi / d \xi=y$ and integrating them along $\Gamma_{16}$ and $\Gamma_{17}$, respectively, it follows that

$$
\begin{gathered}
\int_{\varphi_{6}}^{\varphi} \frac{d s}{\left(s^{2}-\varphi_{7}^{2}\right) \sqrt{\varphi_{6}^{2}-s^{2}}}=\sqrt{-d_{4}}|\xi| \quad\left(\operatorname{along} \Gamma_{16}\right), \\
\int_{0}^{\varphi} \frac{d s}{\left(\varphi_{7}^{2}-s^{2}\right) \sqrt{\varphi_{6}^{2}-s^{2}}}=\sqrt{-d_{4}} \xi \quad\left(\text { along } \Gamma_{17}\right) .
\end{gathered}
$$

Completing the integrals above, we obtain the solutions $\varphi_{16}(\xi)$ and $\varphi_{17}(\xi)$.

(4) When $d_{2}^{2} / 4 d_{4}<d_{1}<0$, the curve $\Gamma_{18}$ possesses the following expression:

$$
\begin{array}{r}
\Gamma_{18}: y^{2}=-d_{4}\left(a_{11}^{2}-\varphi^{2}\right)\left(\varphi^{2}-a_{12}^{2}\right) \varphi^{2}, \\
\text { where } \varphi_{12} \leq \varphi \leq \varphi_{11} .
\end{array}
$$

Substituting (62) into $d \varphi / d \xi=y$ and integrating it along $\Gamma_{18}$, we have

$$
\int_{a_{11}}^{\varphi} \frac{d s}{s \sqrt{\left(a_{11}^{2}-s^{2}\right)\left(s^{2}-a_{12}^{2}\right)}}=\sqrt{-d_{4}}|\xi| \quad\left(\text { along } \Gamma_{18}\right) .
$$

In (63) completing the integral above, we get the solution $\varphi_{18}(\xi)$.

(5) If $d_{1}=0$, the curve $\Gamma_{19}$ possesses the following expression:

$$
\Gamma_{19}: y^{2}=-d_{4} \varphi^{4}\left(-\frac{d_{2}}{d_{4}}-\varphi^{2}\right), \quad \text { where } 0<\varphi \leq \sqrt{-\frac{d_{2}}{d_{4}}} .
$$

Substituting (64) into $d \varphi / d \xi=y$ and integrating it along $\Gamma_{19}$, we get

$$
\int_{\varphi}^{\sqrt{-d_{2} / d_{4}}} \frac{d s}{s^{2} \sqrt{-d_{2} / d_{4}-s^{2}}}=\sqrt{-d_{4}}|\xi| \quad\left(\text { along } \Gamma_{19}\right) .
$$

Similar to the process above, we obtain $\varphi_{19}(\xi)$ and complete the derivations for Proposition 2. Hereto, we have completed the derivations for our main results mentioned in Section 2.

\section{Conclusions}

In this paper, by employing the bifurcation method and qualitative theory of dynamical systems, we study the bifurcations of traveling wave solutions for the Kundu equations (1a) and (1b). In order to derive the traveling wave solutions, we show the bifurcation phase portraits of system (12). Through the phase portraits, we obtain various explicit traveling wave solutions. Also, our work includes some previous results. On the other hand, from some special orbits, we also obtain some new explicit traveling wave solutions.

Note that there are two problems waiting to solve. The first one is that we do not give the elliptic periodic wave solutions for the Kundu equations (1a) and (1b). The relationship between the elliptic periodic wave solutions and the solutions given in this paper is not revealed. The second one is that Zhang et al. [13] showed that the solitary wave solutions $\varphi_{i}(\xi)(i=3,4)$ are of orbital stability. However, we do not know whether the other solitary wave solutions obtained in this paper are of orbital stability.

\section{Acknowledgment}

This research is supported by the National Natural Science Foundation of China (no. 11171115).

\section{References}

[1] A. Kundu, "Landau-Lifshitz and higher-order nonlinear systems gauge generated from nonlinear Schrödinger type equations," Journal of Mathematical Physics, vol. 25, no. 12, pp. 34333438, 1984.

[2] W. van Saarloos and P. C. Hohenberg, "Fronts, pulses, sources and sinks in generalized complex Ginzburg-Landau equations," Physica D, vol. 56, no. 4, pp. 303-367, 1992.

[3] E. Mjolhus, "On the modulational instability of hydromagnetic waves parallel to the magnetic field," Journal of Plasma Physics, vol. 16, no. 3, pp. 321-334, 1976. 
[4] K. Mio, T. Ogino, K. Minami, and S. Takeda, "Modified nonlinear Schrödinger equation for Alfvén waves propagating along the magnetic field in cold plasmas," Journal of the Physical Society of Japan, vol. 41, no. 1, pp. 265-271, 1976.

[5] R. J. Deissler and H. R. Brand, "Generation of counterpropagating nonlinear interacting traveling waves by localized noise," Physics Letters A, vol. 130, no. 4-5, pp. 293-298, 1988.

[6] M. Tsutsumi and I. Fukuda, "On solutions of the derivative nonlinear Schrödinger equation. II," Funkcialaj Ekvacioj, vol. 24, no. 1, pp. 85-94, 1981.

[7] H. Haken, Advanced Synergetics: An Introduction, Springer, Berlin, Germany, 1987.

[8] H. H. Chen, Y. C. Lee, and C. S. Liu, "Integrability of nonlinear Hamiltonian systems by inverse scattering method," Physica Scripta, vol. 20, no. 3-4, pp. 490-492, 1979.

[9] V. S. Gerdjikov and M. I. Ivanov, "A quadratic pencil of general type and nonlinear evolution equations. II. Hierarchies of Hamiltonian structures," Bulgarian Journal of Physics, vol. 10, no. 2, pp. 130-143, 1983.

[10] A. Biswas, "1-soliton solution of the generalized Radhakrishnan, Kundu, Lakshmanan equation," Physics Letters A, vol. 373, no. 30, pp. 2546-2548, 2009.

[11] Z. Feng and X. Wang, "Explicit exact solitary wave solutions for the Kundu equation and the derivative Schrödinger equation," Physica Scripta, vol. 64, no. 1, pp. 7-14, 2001.

[12] D.-J. Huang, D.-S. Li, and H.-Q. Zhang, "New exact travelling wave solutions to Kundu equation," Communications in Theoretical Physics, vol. 44, no. 6, pp. 969-976, 2005.

[13] W. Zhang, Y. Qin, Y. Zhao, and B. Guo, "Orbital stability of solitary waves for Kundu equation," Journal of Differential Equations, vol. 247, no. 5, pp. 1591-1615, 2009.

[14] H. Zhang, "Various exact travelling wave solutions for Kundu equation with fifth-order nonlinear term," Reports on Mathematical Physics, vol. 65, no. 2, pp. 231-239, 2010.

[15] B. L. Guo and Y. P. Wu, "Orbital stability of solitary waves for the nonlinear derivative Schrödinger equation," Journal of Differential Equations, vol. 123, no. 1, pp. 35-55, 1995.

[16] B. He and Q. Meng, "Bifurcations and new exact travelling wave solutions for the Gerdjikov-Ivanov equation," Communications in Nonlinear Science and Numerical Simulation, vol. 15, no. 7, pp. 1783-1790, 2010.

[17] J. Li and Z. Liu, "Smooth and non-smooth traveling waves in a nonlinearly dispersive equation," Applied Mathematical Modelling, vol. 25, no. 1, pp. 41-56, 2000.

[18] Z. Liu and T. Qian, "Peakons of the Camassa-Holm equation," Applied Mathematical Modelling, vol. 26, no. 3, pp. 473-480, 2002.

[19] Z. Liu, R. Wang, and Z. Jing, "Peaked wave solutions of Camassa-Holm equation," Chaos, Solitons \& Fractals, vol. 19, no. 1, pp. 77-92, 2004.

[20] Z. Liu and B. Guo, "Periodic blow-up solutions and their limit forms for the generalized Camassa-Holm equation," Progress in Natural Science, vol. 18, no. 3, pp. 259-266, 2008.

[21] Z. S. Wen, "Extension on bifurcations of traveling wave solutions for a two-component Fornberg-Whitham equation," Abstract and Applied Analysis, vol. 2012, Article ID 704931, 15 pages, 2012.

[22] M. Song, S. Li, and J. Cao, "New exact solutions for the (2+1)dimensional Broer-Kaup-Kupershmidt equations," Abstract and Applied Analysis, vol. 2010, Article ID 652649, 9 pages, 2010.
[23] M. Song, B. S. Ahmed, and A. Biswas, "Topological soliton solution and bifurcation analysis of the Klein-Gordon-Zakharov equation in (1+1)-dimensions with power law nonlinearity," Journal of Applied Mathematics, vol. 2013, Article ID 972416, 7 pages, 2013. 


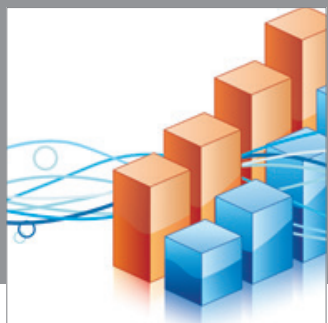

Advances in

Operations Research

mansans

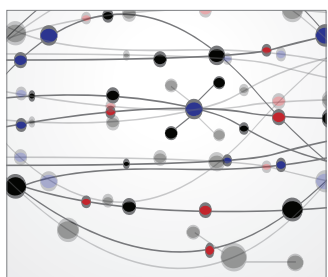

The Scientific World Journal
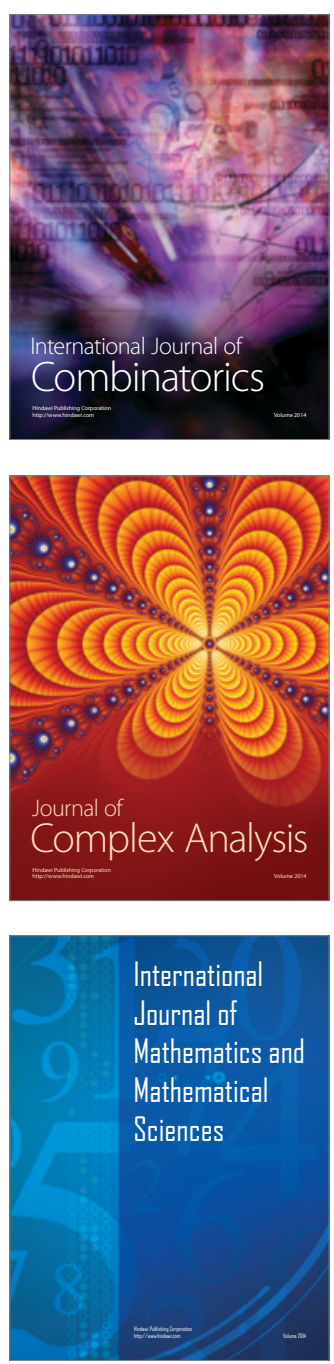
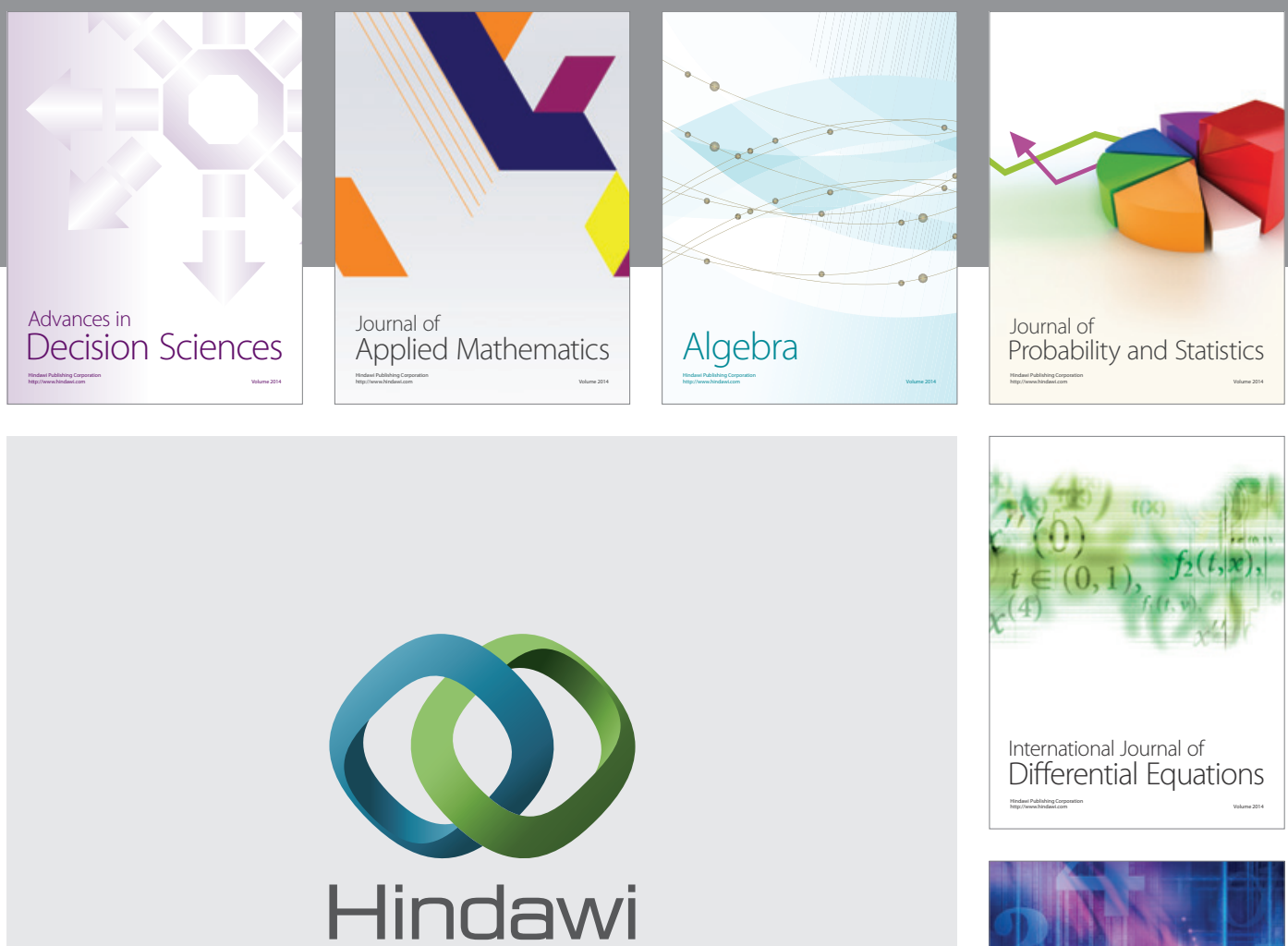

Submit your manuscripts at http://www.hindawi.com
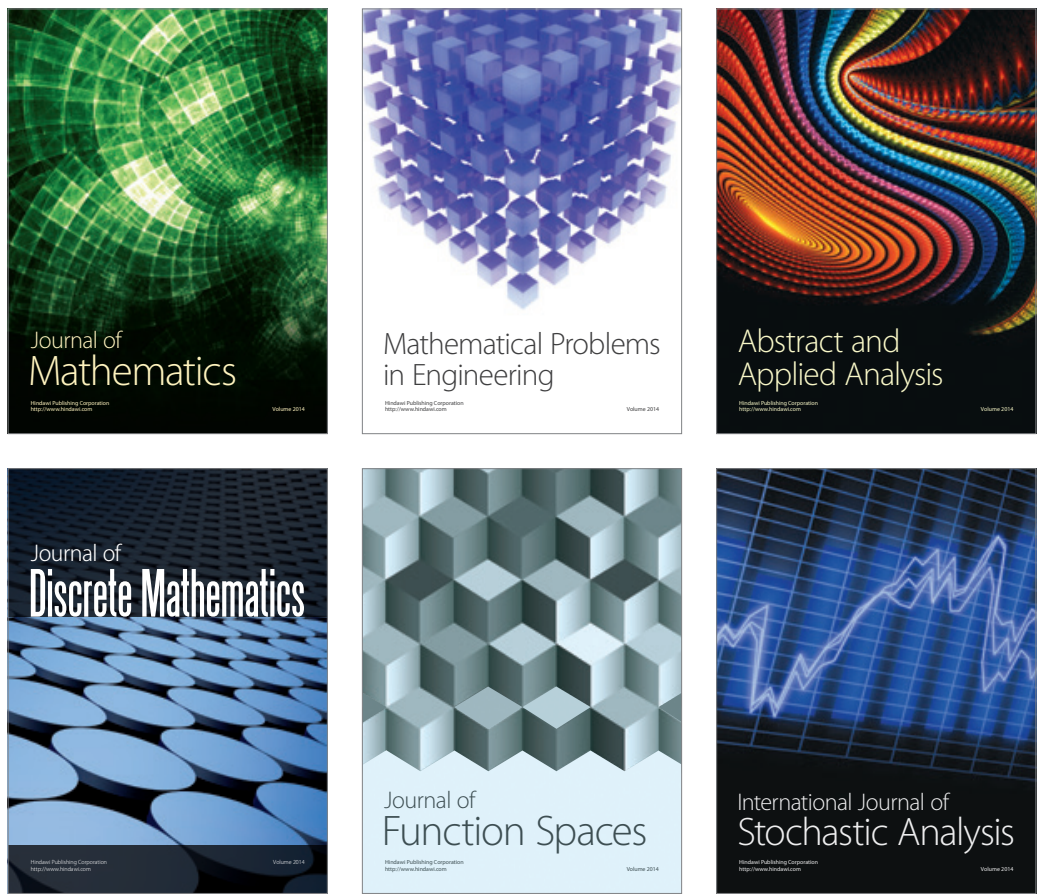

Journal of

Function Spaces

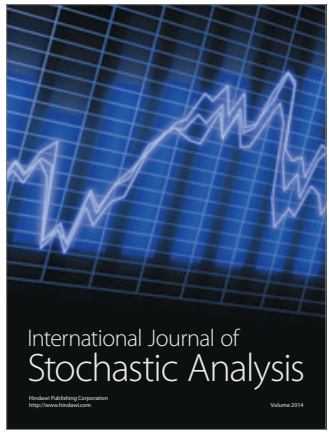

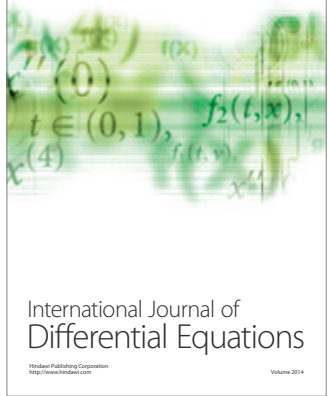
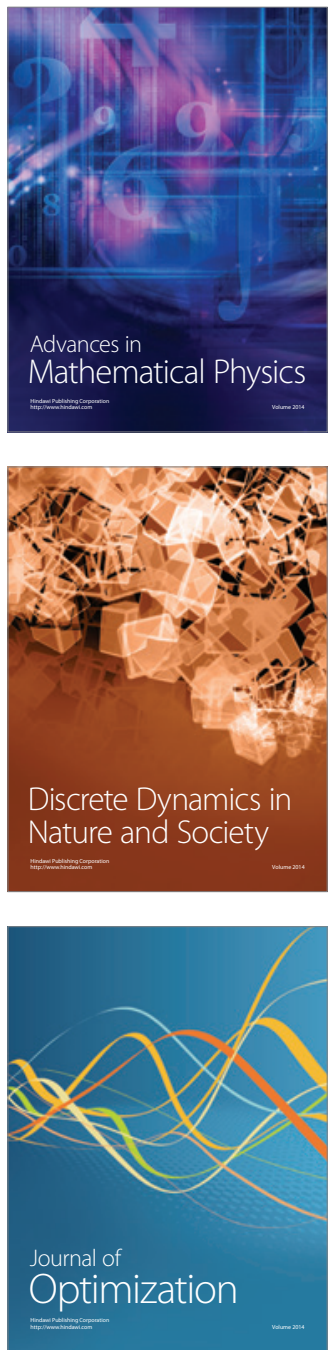